Article

\title{
Optimization Method for Developing Spectral Controlling Cosmetics: Application for Thermal Barrier Cosmetic
}

\author{
Hiroki Gonome ${ }^{1, *(D)}$ and Jun Yamada ${ }^{2}$ \\ 1 Department of Mechanical Systems Engineering, Yamagata University, 4-3-16 Jonan, Yonezawa, \\ Yamagata 992-8510, Japan \\ 2 Department of Mechanical Engineering, Shibaura Institute of Technology, 3-7-5 Toyosu, Koto-ku, \\ Tokyo 135-8548, Japan; jyamada@sic.shibaura-it.ac.jp \\ * Correspondence: gonome@yz.yamagata-u.ac.jp; Tel.: +81-23-826-3103
}

Received: 3 July 2018; Accepted: 11 August 2018; Published: 17 August 2018

\begin{abstract}
In this paper, a method of optimizing a thermal barrier cosmetic and spectral selective cosmetic by controlling the particle size and material is proposed as a countermeasure to heatstroke. The radiative properties of single cosmetic particles of a wide range of particle sizes and wavelengths in non-absorbing air were calculated in this study based on the Mie theory. $\mathrm{Al}_{2} \mathrm{O}_{3}, \mathrm{TiO}_{2}, \mathrm{Au}$, and $\mathrm{Ag}$ were used as the material of the cosmetic particle. The radiative property of a particle cloud in dependent scattering was calculated. The radiative transfer in the cosmetic layer was analyzed, and the spectral reflectance of the cosmetic layer on the human skin was calculated. A new parameter was defined to quantitatively evaluate the performance of the thermal barrier cosmetic and spectral selective cosmetic. For the thermal barrier cosmetic, the $\mathrm{Al}_{2} \mathrm{O}_{3}$ particle was determined to be suitable, and its size was optimized. For the spectral selective cosmetic, the Au particle was likewise determined to be suitable, and its size was optimized. Our cosmetics satisfied both aesthetic and thermal concerns.
\end{abstract}

Keywords: dependent scattering; optimization method; radiative transfer analysis; spectral reflectance; thermal barrier cosmetic

\section{Introduction}

As the problem of global warming has increased, a greater number of heat strokes have been reported. A heatstroke occurs when the summation of the metabolic heat and external heat exceeds an acceptable heat limit of a person. The most significant external heat source that causes a heatstroke is sunlight, especially during outdoor physical activities, such as sports. A thermal barrier cosmetic is therefore proposed in this paper as a countermeasure to heatstroke.

People in outdoor environments are exposed to a considerable amount of sunlight. The solar energy that reaches the Earth's surface through a range from $300 \mathrm{~nm}$ to $2500 \mathrm{~nm}$ is divided into three parts (air mass $=1.5)$ : respective ultraviolet (UV) $(5 \%)$, visible (VIS) $(43 \%)$, and near-infrared (NIR) $(52 \%)$ regions [1]. People absorb large amounts of solar irradiation from the VIS and NIR regions. This absorption increases the body temperature and causes heatstroke.

Cosmetics have a close relation with the light spectrum. Sunscreen was developed to protect human skin from UV light [2]. Depending on the action mechanism, sunscreens can be classified into: physical blockers (i.e., those that reflect the light) and chemical absorbers (i.e., those that absorb the UVA and/or UVB sunlight, subsequently converting it into other forms of energy, preferably at a high rate and yield) [3]. Regarding chemical absorbers, Shaath introduced a simplified qualitative approach suitable for the prediction of the direction of the wavelength shifts in the UV absorption spectrum of a 
sunscreen chemical [3]. In addition, regarding physical blockers, Popov et al. studied the alteration of the optical properties of the superficial layer of human skin at the UV range by the application of nanoparticles of titanium dioxide $\left(\mathrm{TiO}_{2}\right)$, silicon $(\mathrm{Si})$, and zinc oxide $(\mathrm{ZnO})$ [4]. Their theoretical study revealed the optimal sizes of the nanoparticles to minimize the light transmittance of UV wavelengths. However, there is a concern that physical blockers may also contribute to electron transfer reactions and sensitize reactive singlet oxygen. Furthermore, in the VIS region, cosmetics are used to control skin aesthetics. By mimicking the manner in which light interacts with Morpho butterfly scales, while using synthetic materials to form periodic nanostructures, L'Oreal has created a photonic cosmetic product that contains no chemical pigments [5]. Moreover, Yamada et al. numerically investigated radiation transfer in skin by the Monte Carlo method to design and produce cosmetics [6]. Nakamura et al. developed a numerical model to clarify the optical characteristics of the human skin surface and the interaction between the cosmetic particles and human skin [7]. Although NIR radiation comprises $52 \%$ of the energy of sunlight, no cosmetics exist that control NIR radiation. Meanwhile, Gonome et al. developed a spectral selective coating pigmented by nano/microparticles to reflect NIR light for industrial use [8,9]. Álvarez-Docio et al. also developed $\mathrm{CoAl}_{2} \mathrm{O}_{4}$ pigments for NIR light reflection and discussed the effects of the morphology and dispersion of the particles [10]. These spectral selections by means of a scattering medium are also useful for cosmetics.

In this study, two types of thermal barrier cosmetics were theoretically designed. One type-the thermal barrier cosmetic (TBC) - is intended to block all solar heat. Accordingly, the cosmetic reflects sunlight in all solar spectral regions. The other type-the spectral selective cosmetic (SSC) - is intended to maintain the appearance of natural skin, while simultaneously blocking solar heat. Therefore, the reflectance of the cosmetic layer in the VIS region should be similar to that of human skin, and the reflectance in the NIR region should be high.

In the theoretical design of the proposed thermal barrier cosmetics, the radiative property of a single cosmetic particle was calculated. $\mathrm{Al}_{2} \mathrm{O}_{3}, \mathrm{TiO}_{2}, \mathrm{Au}$, and Ag were selected to comprise the material of the cosmetic particle. From these data, the radiative property of a particle cloud in dependent scattering was calculated. Finally, the radiative transfer in the cosmetic layer was calculated, and the effects of the particle sizes and materials on the spectral properties of the cosmetic layer were examined.

\section{Materials and Methods}

\subsection{Complex Refractive Index}

The scattering and absorption of radiation by a single homogeneous spherical particle in a non-absorbing medium can be obtained by solving Maxwell's equations. The radiative properties of a single spherical particle of diameter $d_{p}$, interacting with an electromagnetic wave of wavelength $\lambda$, are governed by two independent non-dimensional parameters, namely the complex refractive index of the particle $m=n-i k$ and the size parameter $x=\pi d_{p} / \lambda$, as described in the Mie scattering theory [11].

Figure 1 shows the spectral distribution of real and imaginary parts of the complex index of refraction of $\mathrm{Al}_{2} \mathrm{O}_{3}, \mathrm{TiO}_{2}, \mathrm{Au}$, and $\mathrm{Ag}$ in the range of 0.3-2.5 $\mu \mathrm{m}$, respectively [12]. The imaginary part of $\mathrm{Al}_{2} \mathrm{O}_{3}$ is almost zero in all spectral regions, and the line is behind the axis. Using the spectral complex refractive index, the radiative properties of a single particle were calculated. 


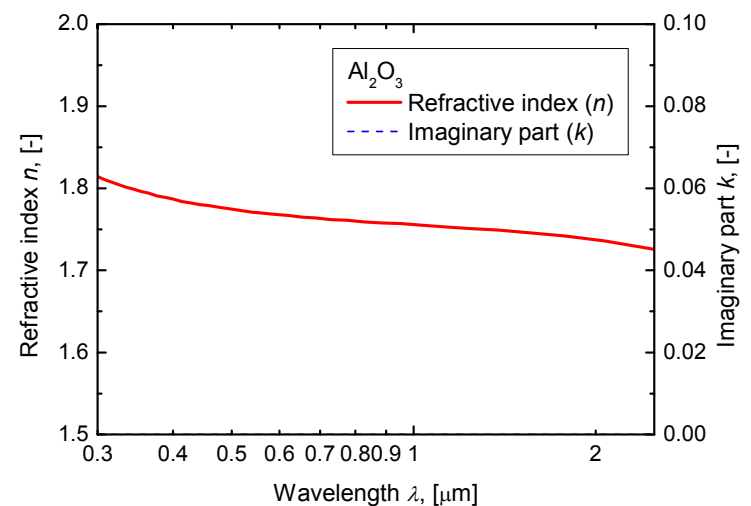

(a)

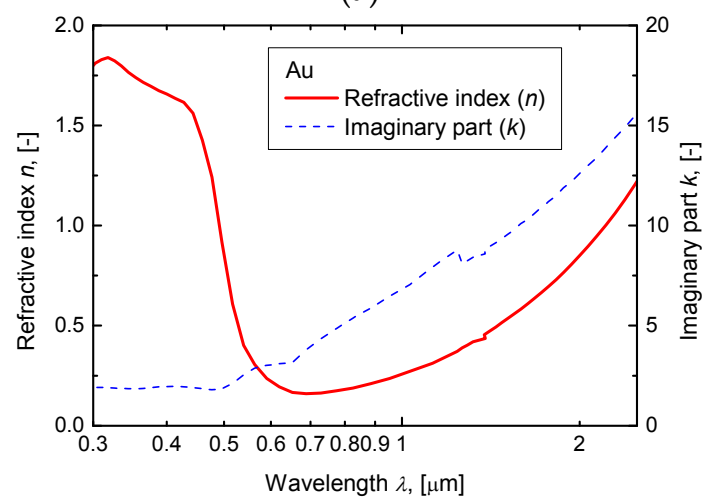

(c)

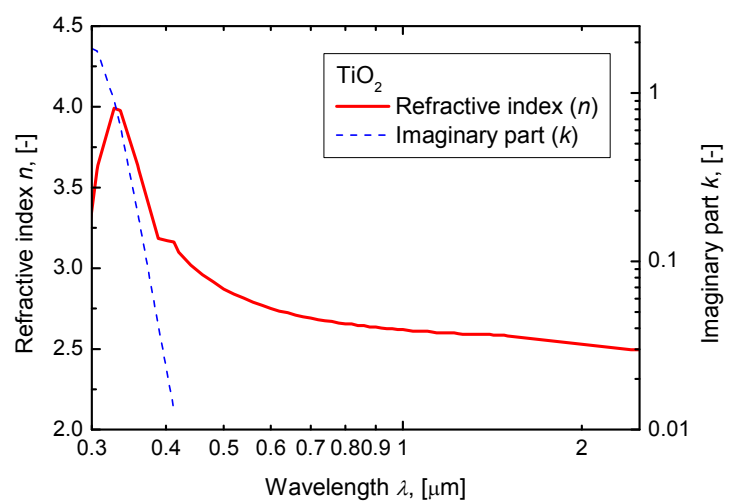

(b)

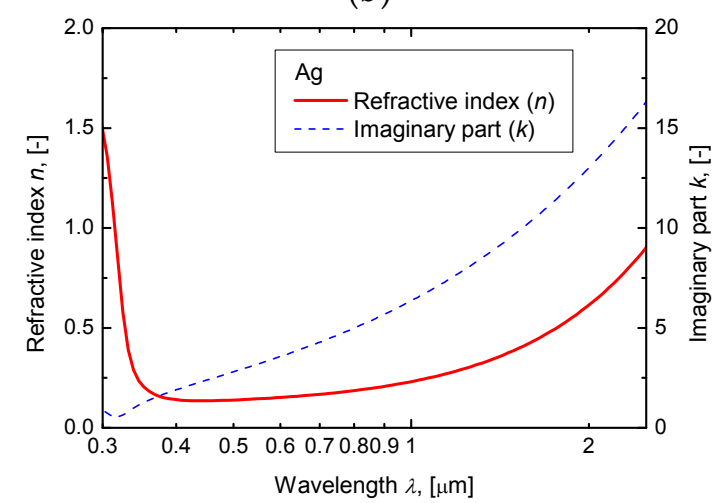

(d)

Figure 1. Spectral complex refractive index $m=n-i k$ : (a) $\mathrm{Al}_{2} \mathrm{O}_{3} ;\left(\right.$ b) $\mathrm{TiO}_{2} ;$ (c) $\mathrm{Au}$; and (d) $\mathrm{Ag}$.

\subsection{Radiative Properties of Single Particles}

According to the Mie theory, the radiative properties of the particle, such as the extinction efficiency factor $Q_{\text {ext }}$ and scattering efficiency factor $Q_{\text {sca }}$, can be calculated using the following equations [13]:

$$
\begin{gathered}
Q_{\text {sca }}=\frac{2}{x^{2}} \sum_{k=1}^{\infty}(2 k+1)\left(\left|a_{n}\right|^{2}+\left|b_{n}\right|^{2}\right) \\
Q_{\text {ext }}=\frac{2}{x^{2}} \sum_{k=1}^{\infty}(2 k+1) \operatorname{Re}\left[a_{n}+b_{n}\right]
\end{gathered}
$$

where $a_{n}$ and $b_{n}$ are the Mie scattering coefficients. The medium is assumed to be non-absorbing with a refractive index equal to 1.0. The particle size varies from 0.01 to $10 \mu \mathrm{m}$ and is logarithmically divided into 100 data points. The radiation interacting with a particle may be scattered from its original direction at angle $\theta$. With the use of the direction $\operatorname{cosine} \mu=\cos \theta$, the scattering phase function $\Phi(\mu)$ can be calculated.

\subsection{Radiative Properties of a Particle Cloud}

\subsubsection{Independent Scattering}

A large collection of particles must be considered in radiative heat transfer through the cosmetic layer. If the scattering is independent, then the effects of a large number of particles are simply expressed as their summation. For simplicity, it is often assumed that a particle cloud consists of spheres that are equally large. The fraction of energy scattered by all particles per unit length along the direction of the incoming beam is equal to the scattering cross-section summed over all the particles. 
If $n_{p}$ is the number of particles per unit volume, and all particles have a uniform diameter $d_{p}$, then the following is true:

$$
\sigma_{s, \lambda, \text { mono }}=\pi\left(\frac{d_{p}}{2}\right)^{2} Q_{\mathrm{sca}} n_{p}, \beta_{\lambda, \text { mono }}=\pi\left(\frac{d_{p}}{2}\right)^{2} Q_{\mathrm{ext}} n_{p}
$$

where $\sigma_{s}$ and $\beta$ are the scattering and extinction coefficients, respectively. Because the phase function in a cloud of uniform particles is the same for each particle, it is also the same for the particle cloud. In this case, the total volume of particles per unit volume or volume fraction can be calculated using the following equation:

$$
f_{v}=\frac{1}{6} \pi d_{p}^{3} n_{p}
$$

\subsubsection{Dependent Scattering}

In a dense cosmetic layer where particles touch, radiation transfer cannot be treated as scattering by separate particles [14]. This effect is referred to as dependent scattering. The relation between independent and dependent scattering is given by following equation [15]:

$$
\beta_{d}=\gamma \beta_{i}, \sigma_{d}=\gamma \sigma_{i}
$$

where scaling factor $\gamma$ is introduced,

$$
\gamma=\frac{1}{1-f_{v}}
$$

here, subscripts $i$ and $d$ indicate independent and dependent scattering, respectively.

A considerable difference arises in the dense cosmetic layer on account of dependent scattering, which is actually a mutual influence of scattering particles. Dependent scattering has no effect on the phase function [16]. Dependent scattering is assumed in our calculations.

\subsection{Radiative Transfer Analysis}

To calculate the radiative transfer in the cosmetic layer, the cosmetic layer is handled as a participating medium in a one-dimensional plane parallel system, as shown in Figure 2. The radiative transfer in this system can be expressed using the one-dimensional radiative transfer equation (RTE):

$$
\frac{1}{\beta_{\lambda}} \frac{d I_{\lambda}(r, s)}{d S}=-I_{\lambda}(r, s)+\frac{\omega_{\lambda}}{4 \pi} \int_{4 \pi} I_{\lambda}\left(r, s^{\prime}\right) \Phi_{\lambda}\left(s^{\prime} \rightarrow s\right) d \Omega
$$

where $I\left(\mathrm{~W} \mathrm{~m}^{-2}(s r)^{-1}\right)$ is the radiative intensity, $r$ is a positional vector, $s$ is a directional vector, $S(\mathrm{~m})$ denotes a path, and $\Omega(s r)$ represents a solid angle. Additionally, $\omega$ is the single scattering albedo and is defined as $\omega=\sigma_{s} / \beta$, which gives the relative importance of scattering.

To solve the RTE, a radiation element method by means of the ray emission model (REM ${ }^{2}$ ) [17] is used. REM ${ }^{2}$ can be applied to anisotropic media with specular and/or diffuse surface boundaries under arbitrary thermal conditions. A one-dimensional plane-parallel system is considered as the analytical model used in REM ${ }^{2}$. Figure 2 shows the analytical model. In this calculation, the dispersed state is assumed to be mono-dispersed. Actually, the particles can make an agglomeration state, and the particle size distribution can change. It affects the spectral radiative properties of the particle cloud $[18,19]$. However, the effect of agglomeration was neglected to simplify the calculation. Elements 1 and $N$ are taken as boundary elements. The $S_{12}$ approximation of Fiveland was used for direction division [12]. A refractive index 1 of air is assumed for all elements. Cosmetics are made of particles and a medium. The medium vaporizes on the skin; then, the cosmetic layer makes the particle layer dense. Therefore, in our calculation, the medium is assumed to be air. However, as indicated in the introduction, there are potential complications of the model due to other processes such as electron or triplet energy transfer in real application. These potential complications are also neglected to simplify the calculation. Solar irradiation enters from element 1 . Spectral solar irradiation is evaluated 
using Bird's model [20]. The basement is human skin, the reflectance of which was measured by Cooksey et al. [21]. The spectral reflectance of human skin is shown in Figure 3.

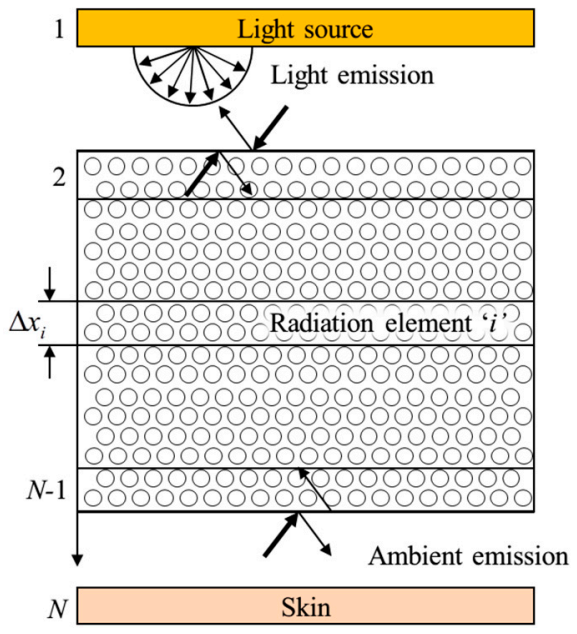

Figure 2. Analytical model for radiative transfer in a cosmetic layer.

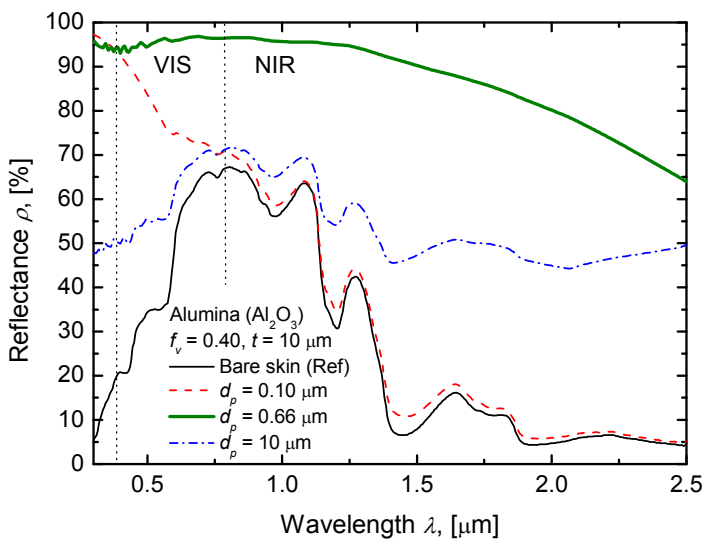

(a)

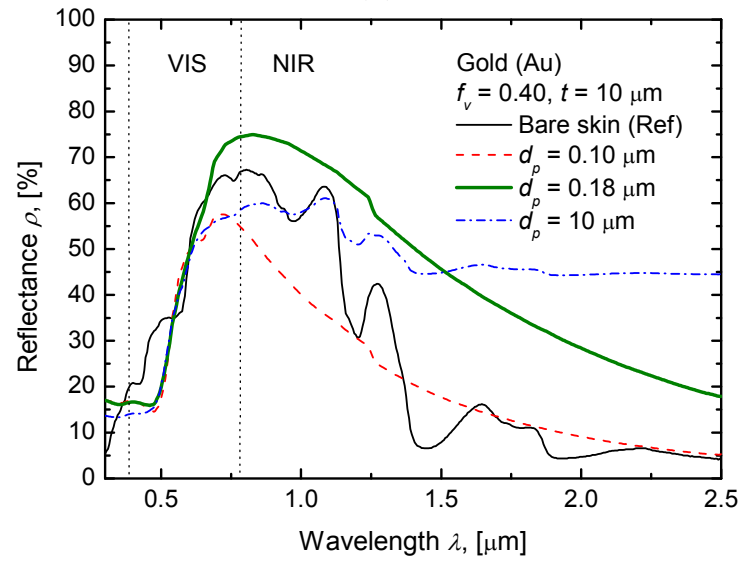

(c)

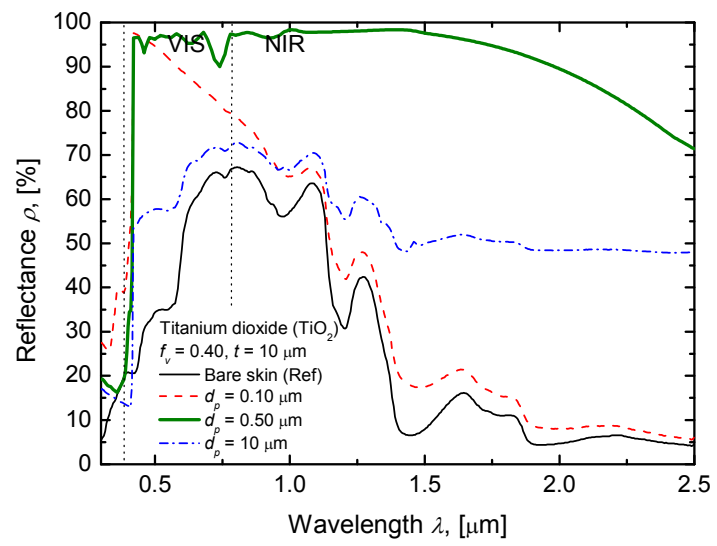

(b)

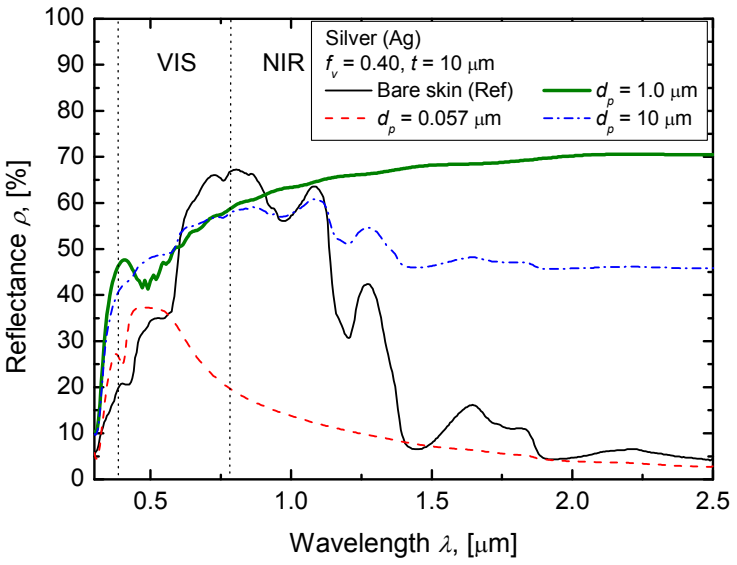

(d)

Figure 3. Calculated spectral reflectance of the cosmetic layer on human skin: (a) $\mathrm{Al}_{2} \mathrm{O}_{3}$; (b) $\mathrm{TiO}_{2}$; (c) $\mathrm{Au}$; and (d) Ag. VIS: visible region; NIR: near-infrared region. 


\subsection{Evaluation Parameter}

The performance parameter of the cosmetic for solar reflectance in the solar spectrum region is proposed as follows:

$$
\rho_{T S R}=\frac{\int_{0.30}^{2.50} \rho(\lambda) I(\lambda) d \lambda}{\int_{0.30}^{2.50} I(\lambda) d \lambda}
$$

where $I(\lambda)$ is the solar irradiation and $\rho(\lambda)$ is the spectral reflectance of the cosmetic layer. This parameter is mainly used for designing TBC and should be high to reflect solar irradiation. A parameter is used to evaluate the maintenance of the visual performance of the cosmetic layer by considering the spectral eye sensitivity. It is defined as follows:

$$
D_{V I S}=\frac{\int_{0.38}^{0.78}\left|\rho(\lambda)-\rho_{\text {skin }}(\lambda)\right| \eta(\lambda) I(\lambda) d \lambda}{\int_{0.38}^{0.78} I(\lambda) d \lambda}
$$

where $\eta(\lambda)$ is the normalized standard luminous efficiency [12]. This parameter should be low for the SSC concept. To design SSC, which maintains the skin's visual appearance and reflects sunlight, the optimization parameter $R$ is defined as follows:

$$
R=\frac{\rho_{T S R}}{D_{V I S}}
$$

To achieve an optimized cosmetic, parameter $R$ should be maximized.

\section{Results and Discussion}

\subsection{Spectral Reflectance}

Figure 3 shows the calculated spectral reflectance of the cosmetic layer on human skin when the volume fraction of the cosmetic particle is 0.40 and the cosmetic layer thickness is $10 \mu \mathrm{m}$. The materials of the cosmetic particle are $\mathrm{Al}_{2} \mathrm{O}_{3}, \mathrm{TiO}_{2}, \mathrm{Au}$, and $\mathrm{Ag}$. For $\mathrm{Al}_{2} \mathrm{O}_{3}$ and $\mathrm{TiO}_{2}$ cosmetics, when the particle diameter is $0.10 \mu \mathrm{m}$, the spectral reflectance peak is in the VIS region, and the spectral reflectance decreases with the wavelength increment. When the particle diameter is an optimum one $\left(\mathrm{Al}_{2} \mathrm{O}_{3}: d_{p}=0.66 \mu \mathrm{m}, \mathrm{TiO}_{2}: d_{p}=0.50 \mu \mathrm{m}\right)$, the spectral reflectance is approximately $95 \%$ of the solar spectrum region. When the particle diameter is $10 \mu \mathrm{m}$, the spectral reflectance increases and comprises approximately $50 \%$ of the NIR region.

These spectral reflectances indicate that $\mathrm{Al}_{2} \mathrm{O}_{3}$ and $\mathrm{TiO}_{2}$ are suitable for TBC. For the Au cosmetic, the spectral reflectance is lower than the ones of bare human skin when the particle diameter is $0.1 \mu \mathrm{m}$. When the particle diameter is $0.18 \mu \mathrm{m}$, the spectral reflectance is higher than those of bare human skin in the NIR spectrum region. At the same time, the cosmetic spectral reflectance is similar to that of bare human skin in the VIS region. In the spectrum over $1.5 \mu \mathrm{m}$, the spectral reflectance of the cosmetic with $d_{p}=10 \mu \mathrm{m}$ is higher than those with $d_{p}=0.18 \mu \mathrm{m}$. However, the solar irradiation in that spectral region is not considerably high; therefore, the thermal barrier performance may not be high. For the Ag cosmetic, the spectral reflectance is lower than the ones of bare human skin when the particle diameter is $0.057 \mu \mathrm{m}$. However, the cosmetic spectral reflectance is similar to that of bare human skin in the short-VIS region. When the particle diameter is over $1.0 \mu \mathrm{m}$, the spectral reflectance is higher than those of bare human skin in the NIR spectrum region. However, the cosmetic spectral reflectance is not similar to that of bare human skin in the VIS region. These spectral reflectances indicate that $\mathrm{Au}$ and Ag are suitable for SSC. 


\subsection{Evaluation Parameter}

\subsubsection{Total Solar Reflectance}

To quantitatively evaluate the thermal barrier performance, the total solar reflectance is calculated according to Equation (8). Figure 4 shows the effect of the particle diameter on the total solar reflectance. For all cosmetics, the total solar reflectance increases with the increasing particle size until it reaches its maximum value. Therefore, when the material of the cosmetic is $\mathrm{Al}_{2} \mathrm{O}_{3}$, the optimum particle for $\mathrm{TBC}$ is 0.614 and $0.658 \mu \mathrm{m}$ when the volume fraction $f_{v}$ is 0.40 and 0.60 , respectively.

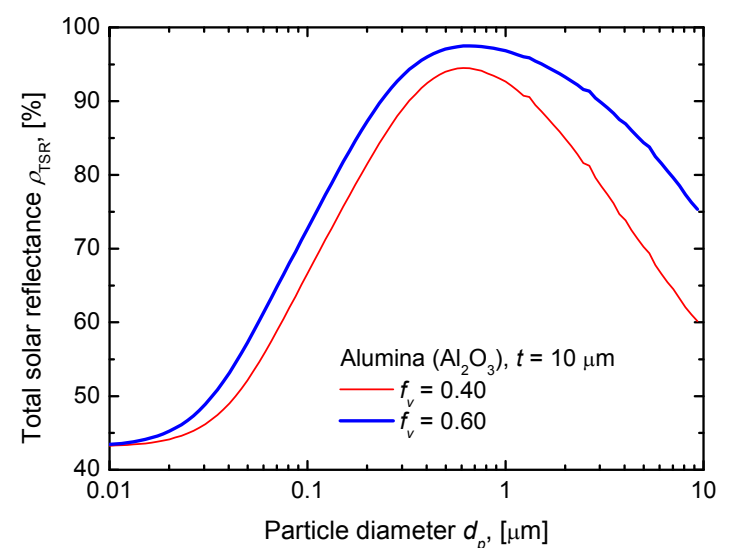

(a)

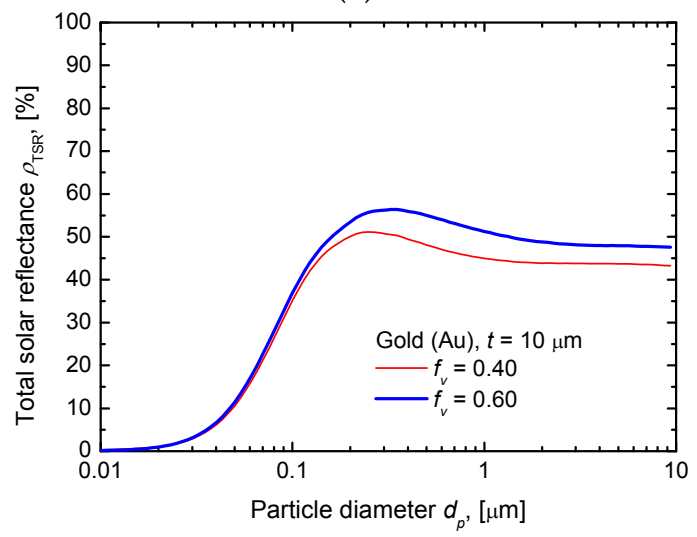

(c)

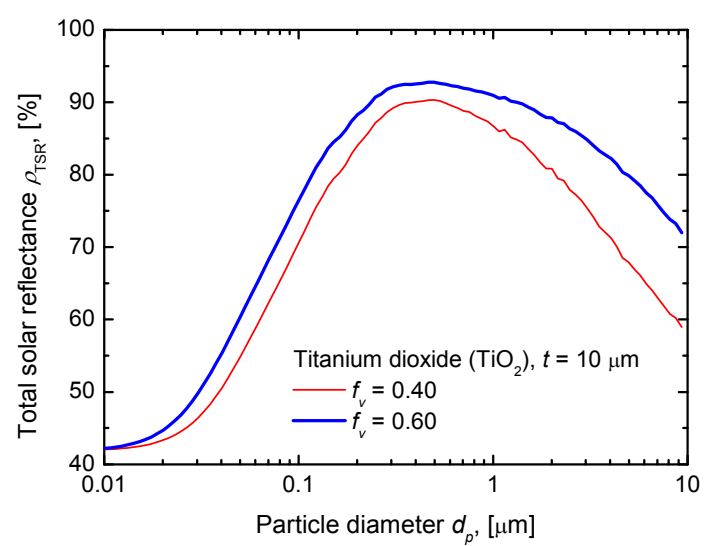

(b)

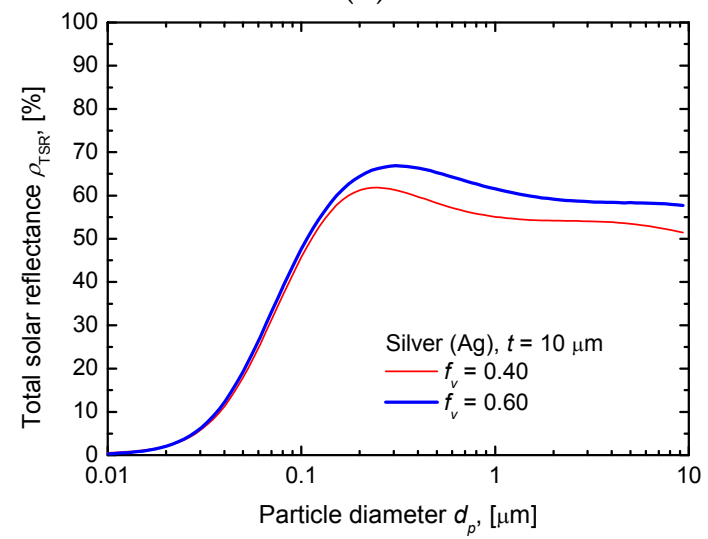

(d)

Figure 4. Particle diameter effect on the total solar reflectance of the cosmetic layer on human skin: (a) $\mathrm{Al}_{2} \mathrm{O}_{3} ;$ (b) $\mathrm{TiO}_{2} ;$ (c) $\mathrm{Au}$; and (d) $\mathrm{Ag}$.

When the material of the cosmetic is $\mathrm{TiO}_{2}$, the optimum particle for $\mathrm{TBC}$ is 0.498 and $0.464 \mu \mathrm{m}$ when the volume fraction $f_{v}$ is 0.40 and 0.60 , respectively. When the material of the cosmetic is Au, the optimum particle for $\mathrm{TBC}$ is 0.248 and $0.327 \mu \mathrm{m}$ when the volume fraction $f_{v}$ is 0.40 and 0.60 , respectively. When the material of the cosmetic is $\mathrm{Ag}$, the optimum particle for TBC is 0.248 and $0.305 \mu \mathrm{m}$ when the volume fraction $f_{v}$ is 0.40 and 0.60 , respectively. When the volume fraction is 0.40 , the maximum total solar reflectance is $94.5 \%, 90.3 \%, 51.2 \%$, and $61.8 \%$ for $\mathrm{Al}_{2} \mathrm{O}_{3}, \mathrm{TiO}_{2}, \mathrm{Au}$, and $\mathrm{Ag}$, respectively. $\mathrm{Al}_{2} \mathrm{O}_{3}$ and $\mathrm{TiO}_{2}$ are so high that they are suitable for TBC, especially $\mathrm{Al}_{2} \mathrm{O}_{3}$. For a large particle diameter, a further increase in the particle diameter decreases the total solar reflectance, and the total solar reflectance converges to its limit value. Finally, we conclude that the $\mathrm{Al}_{2} \mathrm{O}_{3}$ particle with $d_{p}=0.614 \mu \mathrm{m}$ is the optimum particle for TBC. 


\subsubsection{Difference of Visible Reflectance}

To quantitatively evaluate the performance in maintaining the visual quality of skin, the difference of the visible reflectance is calculated according to Equation (9). Figure 5 shows the effect of the particle diameter on the difference of the visible reflectance. For $\mathrm{Al}_{2} \mathrm{O}_{3}$ and $\mathrm{TiO}_{2}$ cosmetics, the difference increases with particle size until it reaches its maximum value. A further increase in particle diameter decreases the difference. To design SSC, the difference should be low; then, the particle diameter is small. However, the total solar reflectance is low when the particle diameter is small, as shown in Figure 4. This result indicates that $\mathrm{Al}_{2} \mathrm{O}_{3}$ and $\mathrm{TiO}_{2}$ are not suitable for SSC.

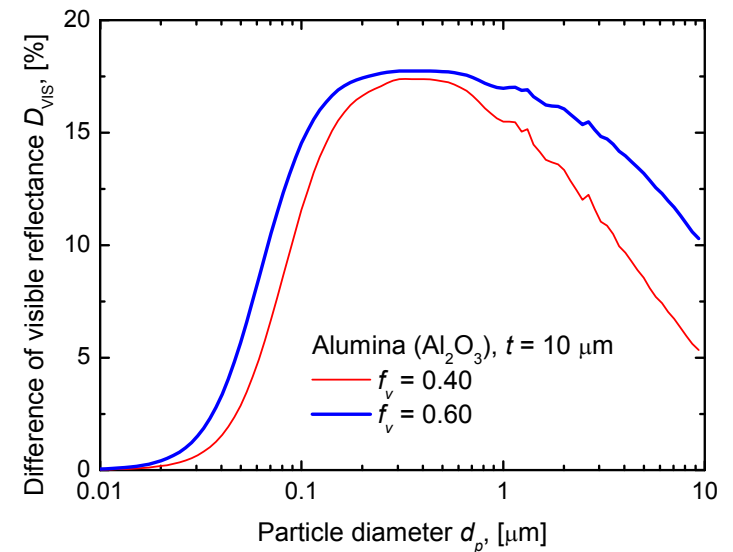

(a)

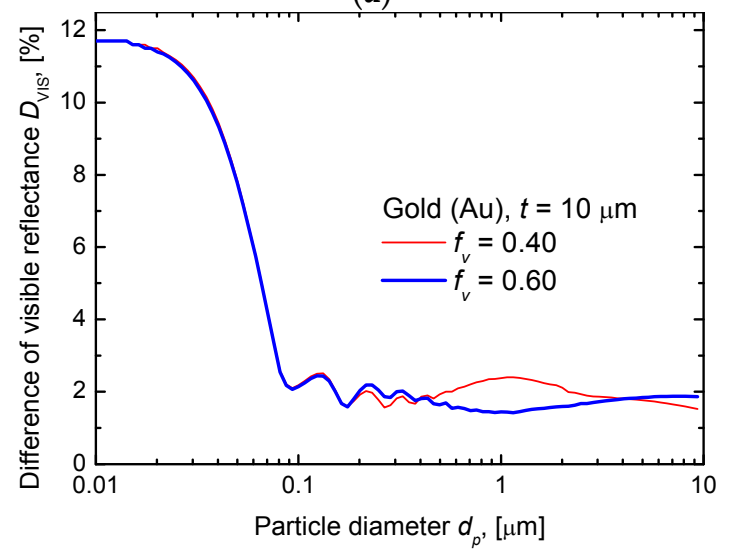

(c)

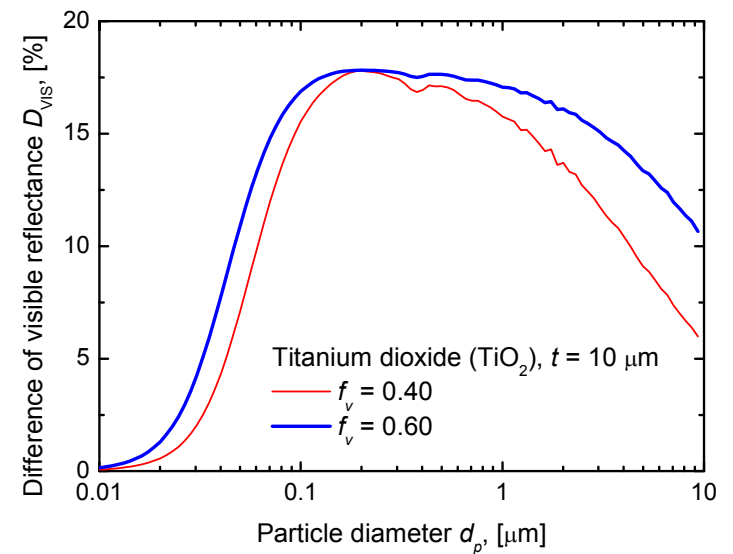

(b)

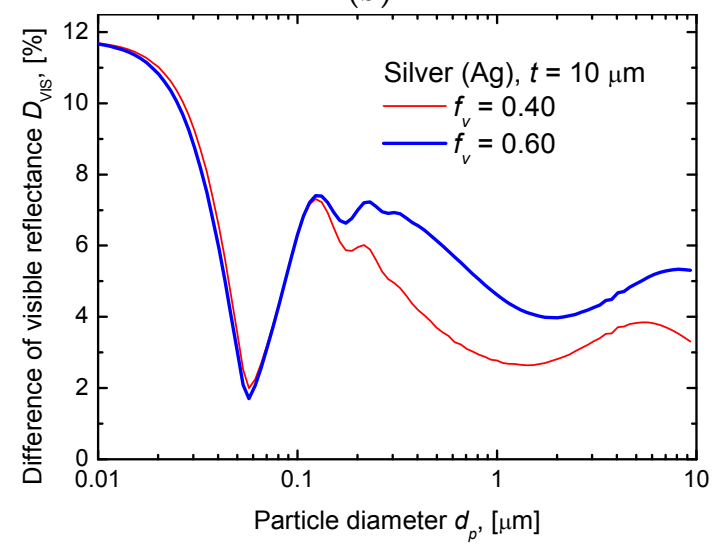

(d)

Figure 5. Particle diameter effect on the difference of visible reflectance of the cosmetic layer on human skin: (a) $\mathrm{Al}_{2} \mathrm{O}_{3}$; (b) $\mathrm{TiO}_{2}$; (c) $\mathrm{Au}$; and (d) $\mathrm{Ag}$.

For the Au cosmetics, the difference decreases with the increasing of the particle size until it reaches its minimum value. A further increase in the particle diameter makes the difference almost the same. The difference of visible reflectance is the lowest in the cosmetic with $9.32 \mu \mathrm{m}$ diameter particles in a 0.40 -volume fraction and with $1.14 \mu \mathrm{m}$ diameter particles in a 0.60-volume fraction. When the difference is small, the total solar reflectance is also high, as shown in Figure 4c. For the Ag cosmetics, the difference decreases with the increasing of the particle size until it reaches its minimum value. A further increase in the particle diameter makes the difference high. The difference of visible reflectance is the lowest in the cosmetic with $0.057 \mu \mathrm{m}$ diameter particles in a 0.40 -volume and a 0.60 -volume fraction. However, when the difference is small, the total solar reflectance is low, as shown in Figure 4d. These results indicate that Au is suitable for SSC. 


\subsubsection{Optimization Parameter}

To quantitatively evaluate the spectral selective performance, optimization parameter $R$ was calculated according to Equation (10). Figure 6 shows the effect of the particle diameter on $R$. $\mathrm{For} \mathrm{Al}_{2} \mathrm{O}_{3}$ and $\mathrm{TiO}_{2}$ cosmetics, the optimization parameter decreases with the increasing of the particle size until it reaches its minimum value. A further increase in the particle diameter increases the optimization parameter. When the optimization parameter is high, the particle diameter is low. The higher optimization parameter corresponds to the smaller particle regime, in which the total solar reflectance is lower Therefore, these cosmetics cannot be used as SSC. However, the difference of the visible reflectance is so small that the optimization parameter becomes high.

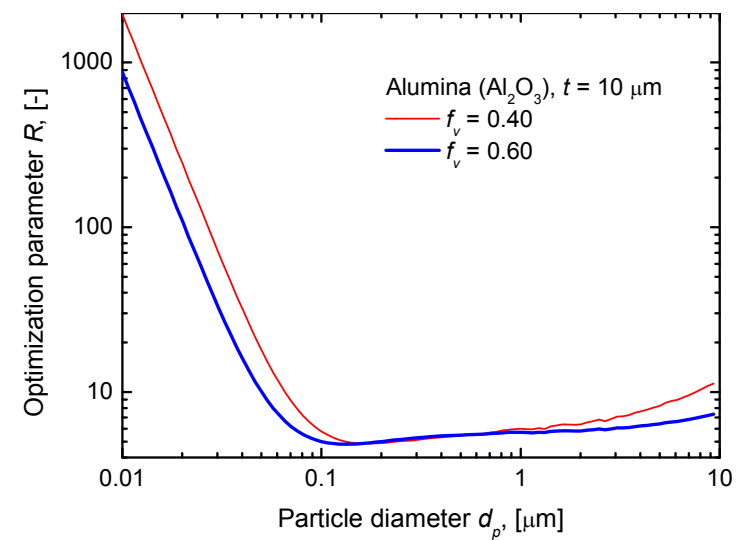

(a)

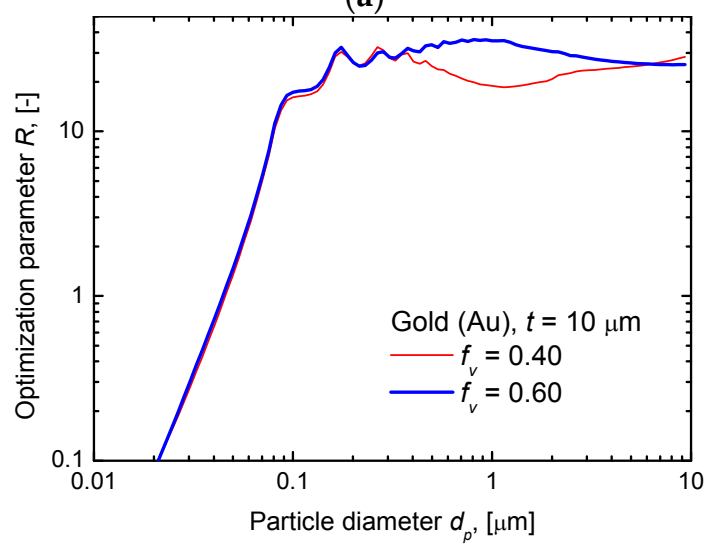

(c)

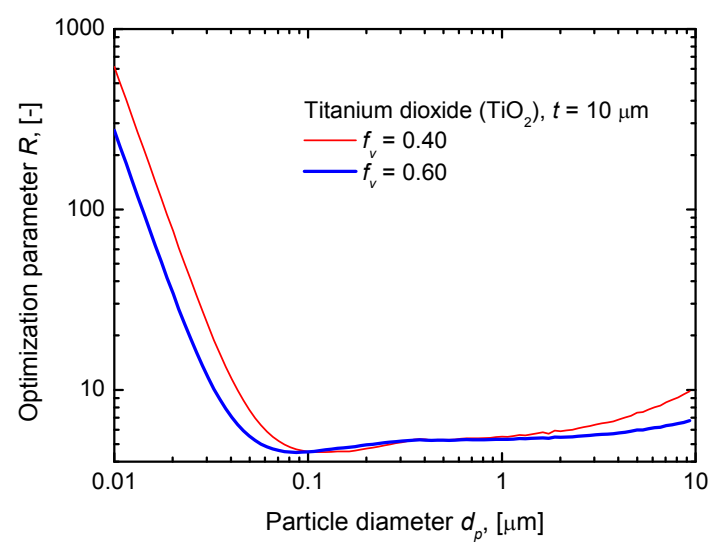

(b)

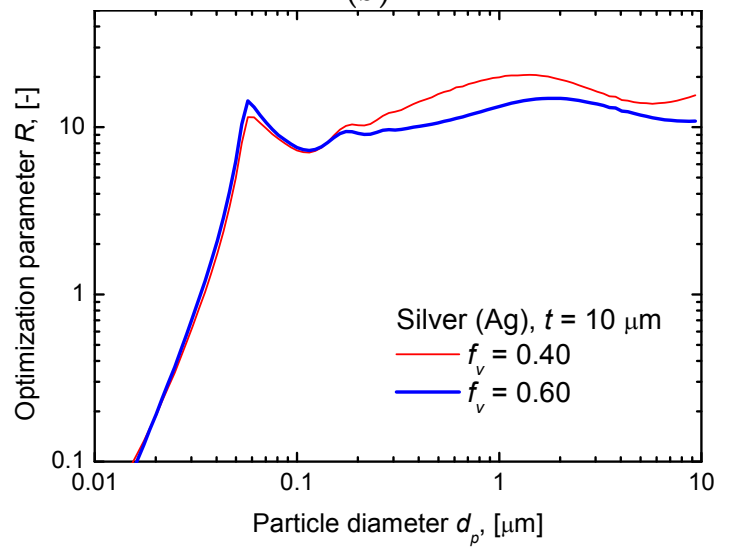

(d)

Figure 6. Particle diameter effect on the optimization parameter of the cosmetic layer on human skin: (a) $\mathrm{Al}_{2} \mathrm{O}_{3} ;$ (b) $\mathrm{TiO}_{2}$; and (c) $\mathrm{Au}$.

For the Au and Ag cosmetics, the optimization parameter increases with increasing the particle size until it reaches its maximum value. A further increase in the particle diameter makes the optimization parameter almost the same. For the Au cosmetics, when the particle diameter is $0.18 \mu \mathrm{m}$, the optimization parameter is approximately 31, regardless of the volume fraction. For the Ag cosmetics, when the particle diameter is $0.057 \mu \mathrm{m}$, the optimization parameter is approximately 14, regardless of the volume fraction. Therefore, we conclude that the Au particle with $d_{p}=0.18 \mu \mathrm{m}$ is the optimum one for SSC. This result shows that the ratio of energy in VIS and NIR regions can help the theoretical design of the spectral selective cosmetic. 


\section{Conclusions}

In this paper, a method of optimizing the thermal barrier cosmetic and spectral selective cosmetic by controlling the particle size and material was proposed. The radiative properties of single cosmetic particles of a wide range of particle sizes and wavelengths in non-absorbing air were calculated based on the Mie theory. $\mathrm{Al}_{2} \mathrm{O}_{3}, \mathrm{TiO}_{2}, \mathrm{Au}$, and $\mathrm{Ag}$ were used as the material of the cosmetic particle. The radiative property of a particle cloud in dependent scattering was calculated. The radiative transfer in the cosmetic layer was analyzed by $\mathrm{REM}^{2}$, and the spectral reflectance of the cosmetic layer on the human skin was calculated. To quantitatively evaluate the spectral selective performance, the three evaluation parameters were defined and the effects of the particle sizes and materials on the spectral properties of the cosmetic layer were discussed.

The spectral reflectance of the cosmetic layer on the human skin was calculated. The new parameter was defined to evaluate the performance of the thermal barrier cosmetic and spectral selective cosmetic. For the thermal barrier cosmetic, the optimum particle was determined to be the $\mathrm{Al}_{2} \mathrm{O}_{3}$ particle with $d_{p}=0.614 \mu \mathrm{m}$. For the spectral selective cosmetic, the optimum particle was determined to be the Au particle with $d_{p}=0.18 \mu \mathrm{m}$. In fact, the cosmetic design should be performed with consideration of the skin sweat gland size, particle mass, and particle cost. However, our optimization method can partly enable the cosmetic design to control the spectral property of the cosmetic. We hope that our designed cosmetic will have beneficial applications, such as helping prevent heatstroke in athletes and workers in outdoor environments.

Author Contributions: Conceptualization, H.G. and J.Y.; Data Curation, H.G. and J.Y.; Formal Analysis, H.G.; Funding Acquisition, H.G.; Investigation, H.G.; Methodology, H.G.; Supervision, J.Y.; Writing-Original Draft, H.G.; Writing-Review \& Editing, H.G.

Funding: This research was funded by the Japan Society for the Promotion of Science KAKENHI (17K14609).

Acknowledgments: We would like to thank Editage (www.editage.jp) for English language editing.

Conflicts of Interest: The authors declare no conflict of interest.

\section{References}

1. ASTM G 173-03 Standard Tables for Reference Solar Spectral Irradiances: Direct Normal and Hemispherical on $37^{\circ}$ Tilted Surface; ASTM International: West Conshohocken, PA, USA, 2012.

2. Diffey, B.; Robson, J. A new substrate to measure sunscreen protection factors throughout the ultraviolet spectrum. J. Soc. Cosmet. Chem. 1989, 40, 127-133.

3. Shaath, N.A. On the theory of ultraviolet absorption by sunscreen chemicals. J. Soc. Cosmet. Chem. 1987, 82, 193-198.

4. Popov, A.P.; Priezzhev, A.V.; Lademann, J.; Myllylä, R. Alteration of skin light-scattering and absorption properties by application of sunscreen nanoparticles: A Monte Carlo study. J. Quant. Spectrosc. Radiat. Transf. 2011, 112, 1891-1897. [CrossRef]

5. Luke, S.M.; Vukusic, P. An introduction to biomimetic photonic design. Europhys. News 2011, 42, 20-23. [CrossRef]

6. Yamada, J.; Kawamura, A.; Miura, Y.; Takata, S.; Ogawa, K. Study on radiation transfer in human skin for cosmetics. J. Quant. Spectrosc. Radiat. Transf. 2005, 93, 219-230. [CrossRef]

7. Nakamura, K.; Ogawa, T.; Takata, S.; Yamada, J. Numerical model for optical characteristics of the human skin surface. Therm. Sci. Eng. 2015, 23, 61-69. (In Japanese)

8. Gonome, H.; Baneshi, M.; Okajima, J.; Komiya, A.; Maruyama, S. Controlling the radiative properties of cool black-color coatings pigmented with CuO submicron particles. J. Quant. Spectrosc. Radiat. Transf. 2014, 132, 90-98. [CrossRef]

9. Gonome, H.; Baneshi, M.; Okajima, J.; Komiya, A.; Yamada, N.; Maruyama, S. Control of thermal barrier performance by optimized nanoparticle size and experimental evaluation using a solar simulator. J. Quant. Spectrosc. Radiat. Transf. 2014, 149, 81-89. [CrossRef]

10. Álvarez-Docio, C.M.; Reinosa, J.J.; del Campo, A.; Fernández, J.F. 2D particles forming a nanostructured shell: A step forward cool NIR reflectivity for $\mathrm{CoAl}_{2} \mathrm{O}_{4}$ pigments. Dyes Pigments 2017, 137, 1-11. [CrossRef] 
11. Mie, G. Beiträge zur Optik trüber Medien, speziell kolloidaler Metallösungen. Annalen der Physik 1908, 330, 377-445. [CrossRef]

12. Palik, E.D. Handbook of Optical Constants of Solids, 1st ed.; Academic Press: Cambridge, MA, USA, 1998.

13. Modest, M.F. Radiative Heat Transfer, 2nd ed.; Academic Press: Cambridge, MA, USA, 2003.

14. Drolen, B.L.; Tien, C.L. Independent and dependent scattering in packed-sphere systems. J. Thermophys. Heat Transf. 1987, 1, 63-68. [CrossRef]

15. Gusarov, A.V.; Kruth, J.P. Modelling of radiation transfer in metallic powders at laser treatment. Inter. J. Heat Mass Transf. 2005, 48, 3423-3434. [CrossRef]

16. Singh, B.P.; Kaviany, M. Modelling radiative heat transfer in packed beds. Int.J. Heat Mass Transf. 1992, 35, 1397-1405. [CrossRef]

17. Maruyama, S.; Aihara, T. Radiation heat transfer of arbitrary three-dimensional absorbing, emitting and scattering media and specular and diffuse surfaces. J. Heat Transf. 1997, 119, 129-136. [CrossRef]

18. Reinosa, J.J.; Docio, C.M.Á.; Ramírez, V.Z.; Lozano, J.F.F. Hierarchical nano ZnO-micro TiO 2 composites: High UV protection yield lowering photodegradation in sunscreens. Ceram. Int. 2018, 44, 2827-2834. [CrossRef]

19. Baneshi, M.; Gonome, H.; Komiya, A.; Maruyama, S. The effect of particles size distribution on aesthetic and thermal performances of polydisperse $\mathrm{TiO}_{2}$ pigmented coatings: Comparison between numerical and experimental results. J. Quant. Spectrosc. Radiat. Transf. 2012, 113, 594-606. [CrossRef]

20. Bird, R.E.; Riordan, C. Simple solar spectral model for direct and diffuse irradiance on horizontal and tilted planes at the earth's surface for cloudless atmospheres. J. Clim. Appl. Meteorolo. 1986, 25, 87-97. [CrossRef]

21. Cooksey, C.C.; Allen, W.D. Reflectance measurements of human skin from the ultraviolet to the shortwave infrared (250 $\mathrm{nm}$ to $2500 \mathrm{~nm}$ ). In Proceedings of the SPIE Defense, Security, and Sensing, Baltimore, MD, USA, 29 April-3 May 2013; p. 87340N.

(C) 2018 by the authors. Licensee MDPI, Basel, Switzerland. This article is an open access article distributed under the terms and conditions of the Creative Commons Attribution (CC BY) license (http:/ / creativecommons.org/licenses/by/4.0/). 